\title{
Design of Picking Robot Manipulator Control System Based on Fuzzy Compensation RBF Neural Network
}

\author{
NaWang ${ }^{1}$, Qinghui Meng ${ }^{1}$, Jie Yang ${ }^{2,3 *}$ \\ ${ }^{1}$ Henan Polytechnic Institute, Nanyang, Henan, China \\ ${ }^{2}$ Henan Institute of Technology, Xinxiang, Henan, China \\ ${ }^{3}$ DivisionofComputerEngineering,DongseoUniversity,Busan, RepublicofKorea \\ *Corresponding Author.
}

\begin{abstract}
Industrial manipulator occupies a very important position in industrial production. The tracking control of its control system and joint trajectory has always been a research hotspot. But the manipulator is a multi input multi output system, which has the characteristics of nonlinearity and strong coupling. Radial basis function (RBF) neural network has high nonlinear mapping ability. In this paper, the structure characteristics, learning algorithm and application of RBF neural network in manipulator control are analyzed. In this paper, the nonlinear approximation property of RBF neural network is theoretically verified. This paper analyzes the basic structure of picking manipulator system in detail. At the same time, the Lagrange Euler method is used to deduce the dynamic equation of the two degree of freedom series manipulator, and the inertia characteristics, Coriolis force and centripetal force characteristics, heavy torque characteristics are analyzed. The nonlinear system model of manipulator based on S-function is established in MATLAB, and the dynamic model is transformed into the form of second-order differential equation to facilitate the introduction of the designed algorithm.
\end{abstract}

Keywords: Industrial manipulator, control system, joint trajectory, tracking control.

\section{Introduction}

The development of the robot field is rapid, but there is still a long way to go from the core mature stage of this field, so up to now, robots cannot let human will, omnipotent. But its role in modern human production and life is irreplaceable, robot technology is imperceptibly integrated into our daily life, become one of us. Moreover, the robot industry chain is gradually becoming complete and has developed into a unique high-tech industry. It can be concluded that with the vigorous development of robot research, robots will not only be applied in industry as they are now, but also infiltrate into ordinary people and become helpers in daily life. The efficiency of industrial production and the quality of people's daily life will be greatly improved.

After decades of efforts, there are many achievements in the control field of industrial robots (manipulators), and there are many control methods. For example, the most classic and mature PID control is still widely used in some simple systems, but it shows poor dynamic performance for complex systems; There are also computational torque control, robust control and so on, which have very good quality in adjusting time and overshoot. The common disadvantage is that they are dependent on the parameters of the mathematical model of the manipulator. They all need an accurate mathematical model of the manipulator, or the uncertainty of the system boundary is given. However, the reality is that the manipulator is accompanied by incomplete information, imprecisely defined parameters and external factors in the operation process [1-3]. These control methods are very sensitive to the lack of sensor information and unplanned accidents, this leads to the traditional model-based robot control method cannot guarantee the robustness of the system in the actual unpredictable environment. Therefore, it is necessary to adopt a new hybrid control method to solve similar problems. Neural network algorithm is widely used in manipulator control, especially in real-time trajectory tracking. This algorithm has strong self-learning characteristics, parallel processing ability, and nonlinear mapping ability and fault tolerance. Combined with other control methods, the designed controller can meet the requirements of manipulator in trajectory tracking.

ISSN: 0010-8189

(C) CONVERTER 2021

Www.converter-magazine.info 


\section{RBF neural network and its characteristics}

The control of manipulator has been a hot topic. Many control methods have been put forward by scholars at home and abroad. Based on the traditional fixed gravity compensation of manipulator, the paper deduces the gravity approximation function of each joint based on the robot space geometry characteristics and torque balance principle. For the right arm of deicing robot, an online gravity compensation control method with small error is designed, which makes it be applied in practice. In the design of manipulator controller in literature [4-6] which is shown in Figure 1, the heavy moment part is unknown. The author uses the empirical method and the test method to set the gravity term as a fixed value to participate in the whole control. The disadvantage is that the tracking accuracy is not high enough, and the control effect of the manipulator is not very ideal for the manipulator such a strong real-time system. Neural network is widely used in manipulator system control because of its special approximation ability.

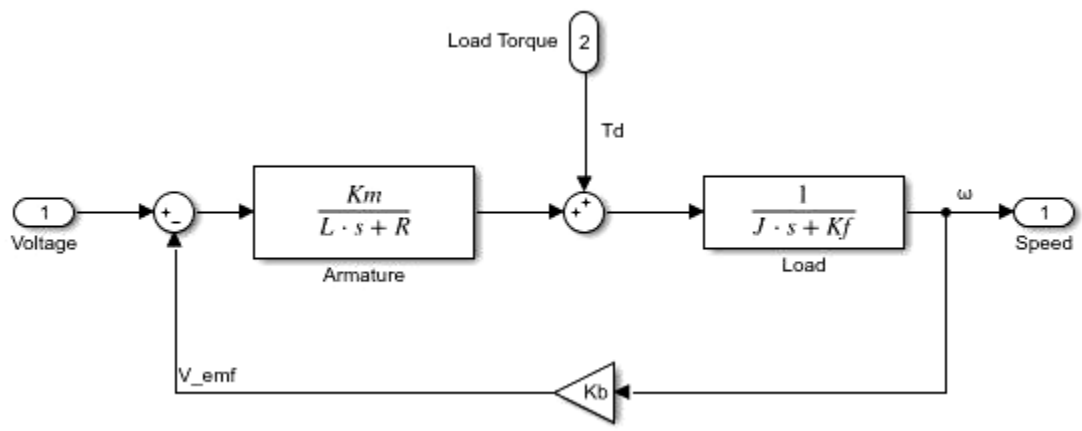

Figure 1. The structure of the manipulator controller in literature [6]

The paper [7] uses global sliding mode surface to combine the nonlinear mapping ability of neural network with the characteristics of sliding mode control. By using RBFNN algorithm and learning, the uncertain boundary of the system is estimated, which weakens the jitter caused by sliding mode control, and achieves certain effect on track tracking, but the initial tracking time has a great fluctuation. In the literature [8], the RBFNN algorithm is combined with T-S fuzzy control to compensate for internal uncertainty, then the dynamic model of manipulator system is established. Then, the trajectory tracking controller is designed by $\mathrm{H} \square$ control method. The simulation results show that the method overcomes the shortcomings of sensitivity to external disturbance and poor tracking performance of maneuvering target. Based on the traditional PD control with error compensation, the paper designs a feedforward controller based on RBFNN algorithm. The controller can learn the output, thus making the feedback input tend to zero, and then cancel the feedback control. Kalman filter is set at the output end to filter the random noise in the system. The control method can increase the side value of random noise and change the sampling frequency of the control system, and can still track the trajectory of the robot quickly. However, the adjustment of the weight of the neural network adopts the method of setting the performance index function, and can not realize the online adaptive weight update. In order to overcome the shortcomings of traditional PD control in manipulator trajectory control, a new control strategy with external interference and uncertainty is proposed. Based on the PD control structure, the radial basis function neural network is introduced to compensate the gravity term of manipulator. The parameters of the network are adjusted by adaptive control law. A robust control term is used to overcome the approximation error of the neural network itself. The stability analysis of the system is carried out by using Lyapunov theory. Finally, the simulation results show that the control algorithm has strong adaptability in convergence speed, tracking accuracy and anti-interference.

The algorithm used in this paper is the radial basis function neural network (RBFNN) algorithm. Pattern recognition technology has led to the development of RBF network. RBF network is widely used in various fields, and it has become the main competitor of multi-layer forward network. Compared with MFN, RBF neural network has better generalization ability, and the network structure is relatively simple, which avoids unnecessary and 
lengthy calculation. The research on RBF network shows that the network can approach any nonlinear function under a compact set. RBFNN is a three-layer forward network. The topology of the RBF network is shown in Figure 2. In Fig. 2, the input layer of the network is composed of parameters known to the actual system. The middle layer is the hidden layer, and the number of neurons is determined according to the actual model. The last layer is output layer, which is mainly based on input and mapping of intermediate layer to get the required amount. The network structure has the characteristics that the information from input space to hidden layer space is nonlinear, while the transmission mode from the hidden layer to the output layer is linear.

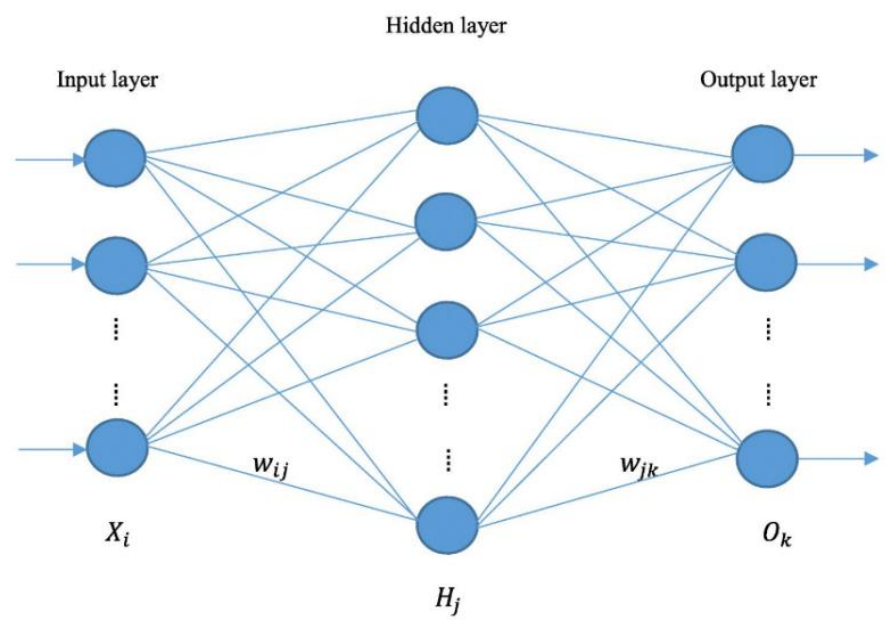

Figure 2. The topology of the RBF network

In this paper, the stability of the system is proved by Lyapunov analysis, which combines the conventional control method with the on-line adaptive RBF neural network control. In the design of manipulator control system, it is an effective method to use RBF neural network to approach the unknown part adaptively. The advantages of neural network are: (1) neural network can use a small amount of prior knowledge to self-study and have the ability of self-learning. This kind of characteristic is a kind of liberation for the complicated mathematical calculation, and it can play a powerful role in the control field involving theoretical calculation. The general control algorithm needs a lot of model prior information to design the control scheme, for example, the mathematical model of the controlled object needs to be established. With the self-learning characteristics, the controller does not need too much model and parameter information of the system. Therefore, neural network control can be widely used to solve the control problem with uncertain model.

(2) Because some single control methods are not effective for nonlinear systems, and there are many design forms of implicit layer transfer function of neural network, which can be used to achieve nonlinear mapping function, so combining neural network provides an effective path for solving nonlinear control problems.

(3) The distributed structure of neural network and parallel computing method make it have the advantage of fault tolerance. Some small number of connection errors have no influence on the whole system.

\section{Trajectory planning algorithm}

We described and expressed the spatial relationship of the two rod pieces of the manipulator, which simplifies the kinematics question into solving the $4 \times 4$ equivalence transformation matrix that connects the ends coordinates system and the fixed reference coordinate system, i.e, the kinematical equation of the robot. Figure 3 shows the structure diagram of the picking robot. Figure 4 is the schematic diagram of the connecting rod coordinate system. 


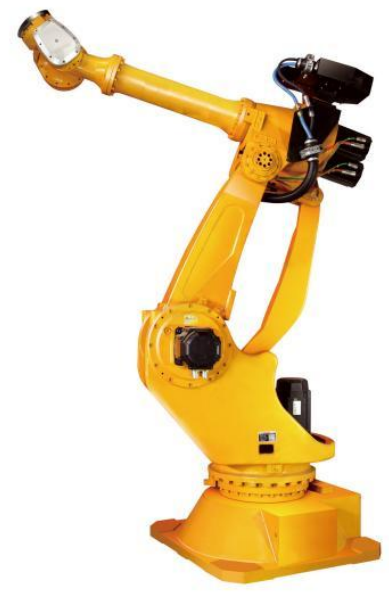

Figure 3. The structure diagram of the picking robot

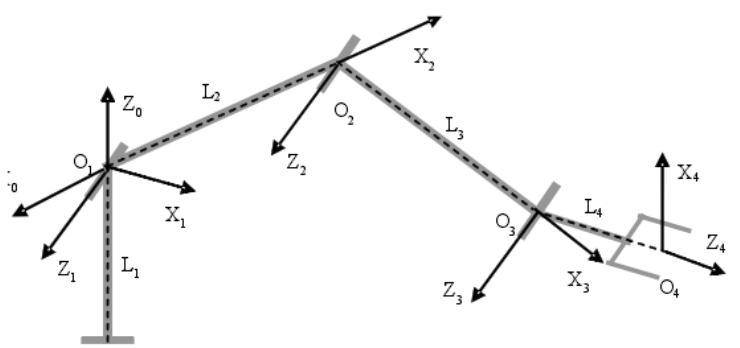

Figure 4. The connecting rod coordinate system

When the spatial relationship between the two adjacent connecting rods i-1 and i changes in accordance with the following motions, the coordinate transformation can be accomplished.

1) Revolve $\theta \mathrm{i}$ around axes $\mathrm{Zi}-1$ until it reaches the position where axes $\mathrm{Zi}-1$ is parallel to axes $\mathrm{Zi}$;

2) Translate distance di along axes $\mathrm{Zi}-1$ to cause $\mathrm{Xi}-1$ to be collinear with $\mathrm{Xi}$

3) Translate distance $\lambda \mathrm{i}$ along $\mathrm{Xi}$ to cause the coordinate system origins of the connecting rods to be coincided;

4) Revolve angle $\lambda \mathrm{i}$ along $\mathrm{Xi}$ to cause axes $\mathrm{Zi}-1$ to be collinear with axes $\mathrm{Zi}$

The basic algorithm is shown as the equation (1) [9-10]:

$$
\begin{gathered}
C^{1}=C-C^{0}, e^{1}=e-e^{0} \\
\eta^{1}=\eta-\eta^{0}, \rho_{1}=\rho-\rho_{0} \\
f(x, \omega)=f^{0}(x, \omega)+\int_{V} S\left(x-x^{\prime}\right)\left(\mathrm{L}^{1} F\left(\mathrm{y}^{\prime}\right)\right. \\
\left.+\rho_{1} \omega^{2} \mathbf{g}(R) \mathrm{T}_{1} f\left(\mathrm{y}^{\prime}\right)\right] S\left(\mathrm{y}^{\prime}\right) \mathrm{dy}^{\prime}
\end{gathered}
$$

ISSN: 0010-8189 
Then we get:

$$
\begin{array}{ll} 
& \frac{1}{\Gamma(1+\alpha)} \int_{R} \frac{f(t)}{(t-x)^{\alpha}}(d t)^{\alpha} \\
& =\lim _{\varepsilon \rightarrow 0}\left[\frac{1}{\Gamma(1+\alpha)} \int_{-\infty}^{x-\varepsilon} \frac{f(t)}{(t-x)^{\alpha}}(d t)^{\alpha}+\right. \\
g_{i k}(\bar{k}, \omega)=-\frac{1}{\eta_{11}^{0}} \frac{1}{\bar{k}^{2}}+\frac{1}{\rho_{0} \omega^{2}}\left(\frac{e_{15}^{0}}{\eta_{11}^{0}}\right)^{2} \frac{\beta_{\perp}^{2}}{\bar{k}^{2}-\beta_{\perp}^{2}} \gamma_{i}\left(\overline{k_{i}}, \omega\right)=\frac{1}{\rho_{0} \omega^{2}}\left(\frac{e_{15}^{0}}{\eta_{11}^{0}}\right)^{2} \frac{\beta_{\perp}^{2}}{\bar{k}^{2}-\beta_{\perp}^{2}} m_{i} & \left.\frac{1+\alpha)^{\alpha}}{(t+\varepsilon}(d t)^{\alpha}\right]
\end{array}
$$

In which,

$$
\begin{array}{r}
\alpha^{2}=\frac{\rho_{0} \omega^{2}}{C_{11}^{0}}, \\
\alpha^{2}=\frac{\rho_{0} \omega^{2}}{C_{66}^{0}} \beta_{\perp}^{2}=\frac{\rho_{0} \omega^{2}}{C_{44}^{\prime}}, \\
C_{44}^{\prime}=C_{44}^{0}+\frac{\left(e_{15}^{0}\right)^{2}}{\eta_{11}^{0}}
\end{array}
$$

Rewrite again Eq. (4) as

$$
\begin{gathered}
\hat{f}_{H}^{\alpha}(x)=\frac{1}{\Gamma(1+\alpha)} \int_{-\infty}^{\infty} \frac{f(t)}{(t-x)^{\alpha}}(d t)^{\alpha} \\
=\frac{1}{\Gamma(1+\alpha)} \int_{-\infty}^{\infty} f(t) g(x-t)(d t)^{\alpha} \\
=f(x) * g(x), \\
\partial_{j}\left(C_{i j k l} \partial_{k} u_{l}+e_{k i j} \partial_{k} \varphi\right)-\rho \ddot{u}_{i}=0 \\
\partial_{j}\left(e_{i j k l} \partial_{k} u_{l}-\eta_{k i j} \partial_{k} \varphi\right)=0
\end{gathered}
$$

\section{Control system design}

The TMS320F2812 chip from TI Company was selected to be the control chip. This chip is a 32-bit fixed-point DSP chip which is suitable for use in industrial control, motor control etc. Its running clock can reach $150 \mathrm{MHz}$, 
and each instruction cycle is 6.67ns. It has 128k x16-bit on-chip FLASH, 18kx16-bit SRAM and abundant peripheral interfaces. In order to reduce the difficulty of the system design, the mature development board QQ2812 was selected. This development board equipped with the F2812 chip and commonly used peripherals and interfaces. Fig. 5 shows the system function block diagram. The communication between the image processing unit and the DSP controller was achieved by RS232 serial port, transmitting the three-dimensional coordinate information of each plucking. According to the position gotten from image processing unit, the DSP can achieve the close-loop control of DC servo motor on $\mathrm{X}, \mathrm{Y}$ and $\mathrm{Z}$ axis and the open-loop control of gripper steer engine.

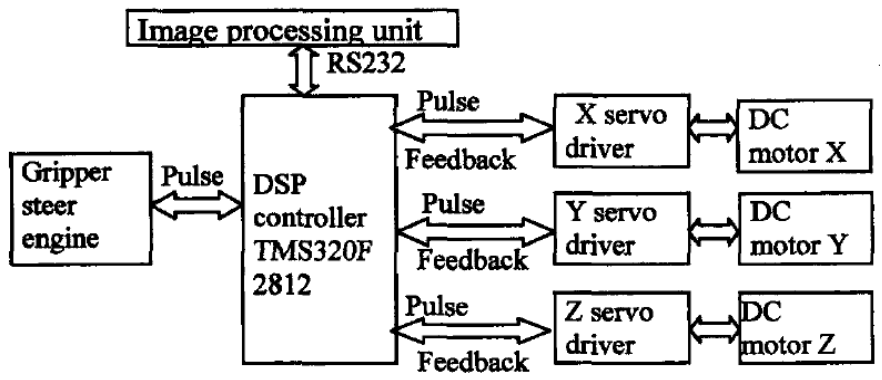

Figure 5. The system function block diagram

This system has 3 DC servo motor whose model is MAX32. Due to the system's mobile power and take energy issues into consideration, a small probability $20 \mathrm{~W}$ DC motor and dear reducer drive program was selected. After the DSP receive the txt data file from image processing unit, the data was specially processed and convert into the position information of the respective axes. Then DSP sent a certain number of pulses to control the motor to rotate a certain angle, so that the each axis can move to the specified location. The motor control system was composed of three control loops which are current loop, velocity loop and position loop. Under the work condition each motor moves according to DSP's construction, and through the three closed loop DSP can achieve high precision velocity control and the position control.

The software was designed by CCS3.3 (Code Composer Studio), using C language and assembly language to achieve all the function. System is initialized after powered on, then steps into the communicating stage, receiving position data from the image processing unit, after which $\mathrm{X}, \mathrm{Y}$ and $\mathrm{Z}$ axis move and the gripper close, picking the tea. Simultaneously, the blower work and the tea are drawn back into the collection box, completing a picking action. Then the system will detect whether all the position have been completed. It will go to next area, if completed. Otherwise, it will go to the next position. After having been developed and debugged, the software was flashed into the FLASH memory of the DSP from the TTAG port via USB emulator. Then it was copied to RAM when working to improve the running speed. After powered on, the system orderly calls concerning functions which contain initializing function, position initializing function-pos_init(), communicating function-data_readQ, coordinate detecting fuction-pos_dectQ, 5 action functions and 3 DSP counter overflow interrupt functions.

The mechanical body is the execution unit of the picking robot whose performance index has immediate impact on the quality of the operation task. The mechanical performance index of the robot, which reflects the tasks competent for the robot and the operability it possesses, is determined by the factors such as accuracy of mechanical manufacture, the joint clearance of the transmission bending deflection, kinematics error of the joint servo motor, and the control system performance.

The performance index can be evaluated from various aspects like kinematics, dynamics and control methods. Among which, kinematics performance has the biggest influence on the operation performance of the vegetablefruit picking robot.

Test of the forward kinematics solution. The forward kinematics solution for the 4 DOF picking robot is 
theoretically solved according to D-H transformation of the robot joint coordinate system. Experiments are done to test and verify the correctness of the forward kinematics solution and the robot performance. Six groups of the joint variables are chosen randomly from the robot joint space. The robot starts motion from zero point. The actual positions of the manipulator in the robot base coordinate frame are measured. The six groups of the joint variables are substituted into the forward kinematics solution equation to obtain the theoretical position. The test result of the forward kinematics solution can be obtained with actual position compared with the theoretical position.

Matlab is a high-level language with high efficiency used in scientific engineering calculation. It is also good at visual display of data in that it is capable of showing data's two, three or even four dimensional manifestations. Fig. 6 and Fig.7 show respectively the picking robot's working space drawn with Matlab's three dimensional plotting function plot 3 and its projection on Plane xoz.

The result of calculation shows that the robot's working space $\mathrm{V}$ equals $1.92 \mathrm{~m} 3$, and the volume index reaches 0.64 . The simulation results prove such designed robot meets the expectations for eggplant picking in greenhouse and is able to work more efficiently. The simulation validates the rationality of the structure design

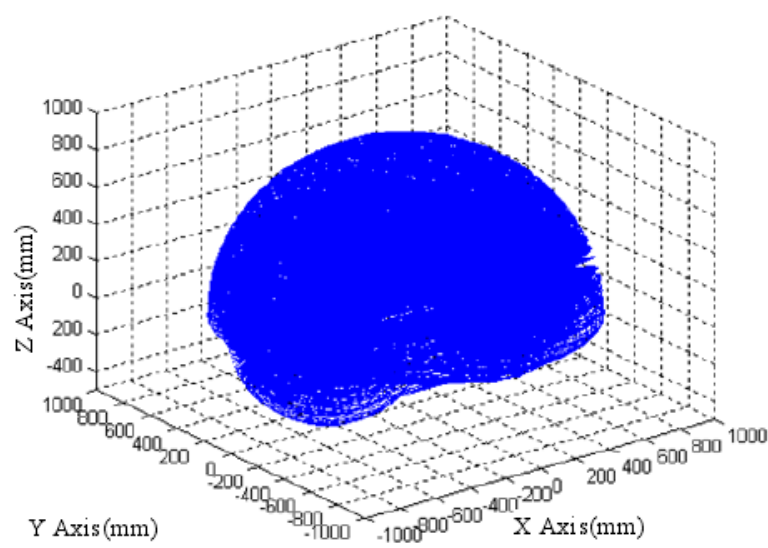

Figure 6. The working space for picking robot

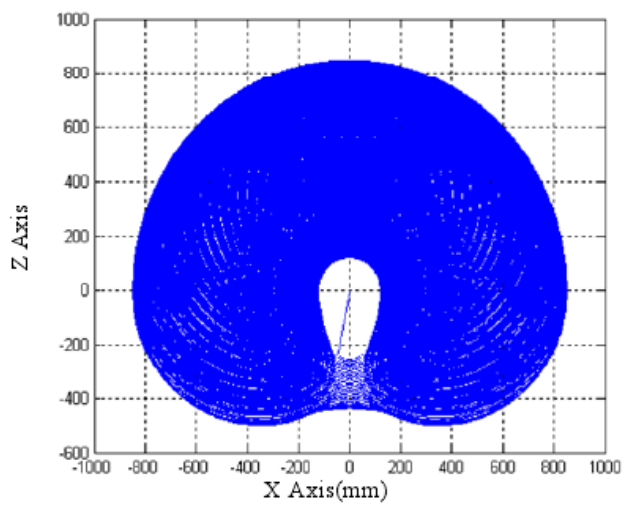

Figure 7. The projection of the working space on plane xoz

\section{Conclusion}

Everything has two sides. To strengthen the innovation of College Students' Education in the environment of we media, we must face the challenges and seize the good development opportunities, continue to ensure the effectiveness of College Students' education, and ensure the realization of the fundamental task of education that

ISSN: 0010-8189 
college students become socialist builders and successors. Based on the existing research results, this paper analyzes the meaning, characteristics and significance of College Students' educational innovation in we media environment, and summarizes the current situation, opportunities and challenges of College Students' educational innovation in we media environment combined with empirical research. On this basis, this paper discusses the goals, principles, methods and methods of College Students' educational innovation in we media environment Content and approach.

\section{Acknowledgements}

This research was supported by the Key Scientific Study Projects of Higher Education Institutions of Henan Province (Grant No. 20B470003), the Promotion Special Project of the Scientific Study Program of Henan Province (Grant No. 202102210084).

\section{References}

[1] Zhang, Tiezhong, and B. Lin. "Object extraction for the vision system of fruit picking robot." Journal of China Agricultural University 9.2(2004):68-72.

[2] $\mathrm{Gu}, \mathrm{B}$., et al. "Design and experiment of intelligent mobile fruit picking robot." Nongye Jixie Xuebao/transactions of the Chinese Society of Agricultural Machinery 43.6(2012):153-160.

[3] Wei, Xiangqin, et al. "Automatic method of fruit object extraction under complex agricultural background for vision system of fruit picking robot." Optik - International Journal for Light and Electron Optics 125.19(2014):5684-5689.

[4] Fu, Xiao Jin, et al. "The Fruit-Picking Robot." Applied Mechanics \& Materials 610(2014):515-518.

[5] Chiu, Yi Chich, S. Chen, and J. F. Lin. "Study of an Autonomous Fruit Picking Robot System in Greenhouses *." Engineering in Agriculture Environment \& Food 6.3(2013):92-98.

[6] Ji, Wei, et al. "Compliance grasp force control for end-effector of fruit-vegetable picking robot." Transactions of the Chinese Society of Agricultural Engineering 30.9(2014):19-26.

[7] Zhao, Dong Hui, H. Zhang, and J. X. Hou. "Design of Fruit Picking Device Based on the Automatic Control Technology." Key Engineering Materials 620(2014):471-477.

[8] Peng, Hongxing, et al. "Fruit image segmentation based on evolutionary algorithm." Nongye Gongcheng Xuebao/transactions of the Chinese Society of Agricultural Engineering 30.18(2014):294301.

[9] $\quad \mathrm{Fu}, \mathrm{L}$., et al. "Development and experiment of end-effector for kiwifruit harvesting robot." Nongye Jixie Xuebao/transactions of the Chinese Society of Agricultural Machinery 46.3(2015):1-8.

[10] Schuetz, C., et al. "Evaluation of a direct optimization method for trajectory planning of a 9-DOF redundant fruit-picking manipulator." International Telecoms Sync Forum Itsf 2015.5(2015):26602666. 